\title{
Funcionalidad del tobillo asociada a la colocación de bota ortopédica neumática en pacientes con fractura de maléolo lateral aislado tipo Weber B estables
}

\author{
Ankle functionality associated with use of pneumatic orthopedic boot in \\ patients with stable Weber $B$ type isolated lateral malleolus fracture
}

\section{Carla Lorena Orozco Álvarez*}

*Especialista en Traumatología y Ortopedia de la Universidad de Guadalajara. Alta Especialidad en Cirugía de la Mano de la Universidad Autónoma de Guadalajara. Diplomado en Plexo Braquial y Lesiones Nerviosas Periféricas de la Universidad Autónoma de Coahuila. México.

\begin{abstract}
Resumen
Las fracturas de tobillo son una de las lesiones óseas de extremidades más comunes en todas las edades, con un predominio de hasta $75 \%$ en etapa productiva. Las fracturas de maléolo lateral aislado representan un porcentaje significativo de las mismas y el tratamiento quirúrgico es el habitual. El objetivo de este estudio es optar por un algoritmo de tratamiento no quirúrgico en aquellos pacientes con fracturas de maléolo lateral aislado tipo Weber B estables con bota ortopédica neumática y analizar los resultados funcionales a corto plazo. Material y métodos: Estudio transversal analítico, realizado de agosto de 2018 a agosto de 2019 en pacientes con fractura de maléolo lateral aislado tipo Weber B tratados con colocación de bota ortopédica neumática tipo Walker larga y seguimiento con radiografías de tobillo con descarga de peso en cuatro ocasiones (una, dos, seis y 12 semanas) para evaluar la alineación del trazo fracturario, la modificación de la medición del claro medial y el proceso de consolidación ósea. Las mediciones de las escalas funcionales OMA, FAAM y AOFAS se realizaron por un médico estandarizado a los 12 meses del evento y los resultados se compararon con un grupo control tratado de manera quirúrgica. Resultados: Se incluyeron 44 pacientes, 26 hombres (59.1\%) y 18 mujeres (40.9\%). La media de edad fue de 34.7 años. Veintitrés pacientes $(52.2 \%)$ fueron tratados con bota y $21(47.7 \%)$ de manera quirúrgica. En cuanto a la valoración de escalas funcionales, para AOFAS, la media de puntuación fue de 89.6 puntos, catalogando $95.5 \%$ de la muestra como funcional $(n=42)$. La media de puntuación de FAAM fue de 80.6 puntos y OMA de 93.4 puntos. Destacan la asociación estadísticamente significativa entre la intervención quirúrgica y la inestabilidad del retropié por AOFAS (ro $=0.499, p=0.001$ ). Así como mayor dolor residual en aquéllos con intervención quirúrgica. Conclusión: La colocación de bota ortopédica neumática se asocia a buena funcionalidad del tobillo en pacientes con fractura de maléolo lateral aislado tipo Weber B estable.
\end{abstract}

Palabras clave: Bota ortopédica, bota Walker, fractura de tobillo, Weber B, fractura de maléolo lateral, fractura peroné,

\begin{abstract}
Ankle fractures are one of the most common limb bone injuries of all ages, with a prevalence up to $75 \%$ in the productive stage. Isolated lateral malleolus fractures represent a significant percentage of them, with surgical treatment being the usual one. The objective of this study is to opt for a non-surgical treatment algorithm in patients with stable Weber B type isolated lateral malleolus fractures using a pneumatic orthopedic boot and to analyze short-term functional results. Material and methods: Analytical cross-sectional study conducted from August 2018 to August 2019 in patients with Weber B type isolated lateral malleolus fracture treated with a long Walker-type pneumatic orthopedic boot and follow-up with weight-bearing ankle X-rays on four occasions $(1,2,6,12$ weeks) to evaluate fracture line alignment, modification of ankle medial clear space and bone healing process. Measurements of OMA, FAAM and AOFAS functional scales were performed by a standardized physician 12 months after the event and the results were compared with a control group treated surgically. Results: 44 patients were included, 26 men (59.1\%) and 18 women (40.9\%). The mean age was 34.7 years. 23 patients (52.2\%) were treated with a boot and 21 (47.7\%) were treated surgically. Regarding the assessment of functional scales; for AOFAS the mean score was 89.6 points, classifying $95.5 \%$ of the sample as functional $(n=42)$. The mean FAAM score
\end{abstract}

Correspondencia:

Carla Lorena Orozco Álvarez

E-mail: carlaa.orozco@ hotmail.com

Citar como: Orozco ÁCL. Funcionalidad del tobillo asociada a la colocación de bota ortopédica neumática en pacientes con fractura de maléolo lateral aislado tipo Weber B estables. Orthotips. 2022; 18 (1): 33-40.

Recibido: 19-09-2021. Aceptado: 06-10-2021. https://dx.doi.org/10.35366/103730 
was 80.6 points and the mean OMA score was 93.4 points. The statistically significant association between the surgical intervention and the instability of the hindfoot in the AOFAS scale stands out ( $r O=0.499, p=0.001)$. As well as, greater residual pain in those with surgical intervention. Conclusion: Pneumatic orthopedic boot use is associated with good ankle functionality in patients with stable Weber $B$ type isolated lateral malleolus fracture.

Keywords: Orthopedic boot, Walker boot, ankle fracture, Weber B, lateral malleolus fracture, fibula fracture.

Abreviaturas:

$O M A=$ Olerud molander ankle score.

$F A A M=$ Foot and Ankle Ability Measure.

AOFAS = American Orthopaedic Foot and Ankle Society.

$E V A=$ Escala visual análoga

\section{Introducción}

Las fracturas de tobillo son una de las lesiones óseas de extremidades más comunes en todas las edades, con un predominio de hasta $75 \%$ en etapa productiva. ${ }^{1}$ Las fracturas de maléolo lateral aislado representan un porcentaje significativo de las mismas. Su etiología, en la mayoría de los casos, es secundaria a un traumatismo indirecto de baja energía ocasionado con frecuencia en la práctica deportiva o en las actividades de la vida diaria. ${ }^{2}$

En 1950 Lauge-Hansen publicó una clasificación que correlaciona el trazo fracturario con el mecanismo de lesión. Por otra parte, en 1965 Weber modificó la clasificación de Danis (Figura 1) haciendo hincapié en la altura del trazo fracturario en el peroné para así evidenciar lesiones ligamentosas a través de la sindesmosis. En la actualidad, ambas clasificaciones son las más utilizadas y ayudan al cirujano ortopedista a describir la lesión y dictar el manejo de la misma.
La clasificación de Lauge-Hansen (Figura 2) describe tanto la posición del pie (pronación o supinación) como la dirección de la fuerza deformante (rotación externa, abducción o aducción). Este estudio centra su atención únicamente en el mecanismo de supinación y rotación externa; es importante conocer que existen cuatro etapas en este tipo de patrón y que con cada una de éstas aumenta la severidad de la lesión y el grado de complejidad del tratamiento. La tipo I involucra la ruptura del ligamento tibioperoneo anterior, mientras que la tipo II involucra una fractura oblicua del peroné o ruptura del complejo ligamentario lateral del tobillo. Ambos tipos se consideran estables y se tratan de manera conservadora. En el patrón tipo III hay ruptura del ligamento tibioperoneo posterior o fractura del maléolo posterior; por último, el tipo IV implica una fractura del maléolo medial o ruptura del ligamento deltoideo.

Por lo tanto, se puede interpretar que el involucro medial, ya sea por fractura del maléolo medial o ruptura del ligamento deltoideo implica inestabilidad de la articulación del tobillo y se debe reparar quirúrgicamente; por lo cual es importante diferenciar entre las fracturas Lauge-Hansen tipo II y tipo IV.

Si existe ruptura del ligamento deltoideo, será difícil de identificar, ya que es clínicamente inespecífico (dolor,
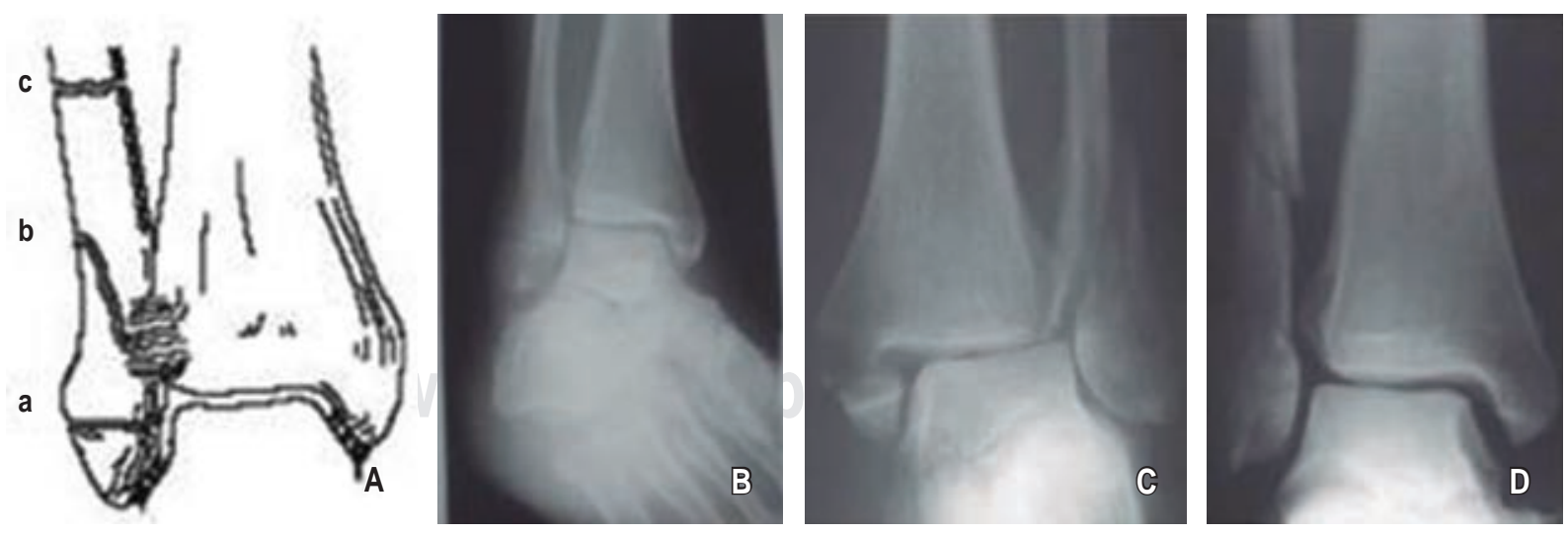

Figura 1: A) Esquema de la clasificación de Danis-Weber de las fracturas de tobillo. B) Fractura tipo A. C) Fractura tipo B. D) Fractura tipo C. Clasificación de la AO/OTA (Arbeitsgemeinschaft für Osteosynthesefragen -Asociación para el Estudio de la Osteosíntesis- y Orthopaedic Trauma Association -Asociación de Trauma Ortopédico-) de las fracturas de tobillo.

Fuente: Bucholz RW ed. Rockwood \& Green's. Fracturas en el adulto. Madrid: Marbán; 2003. 

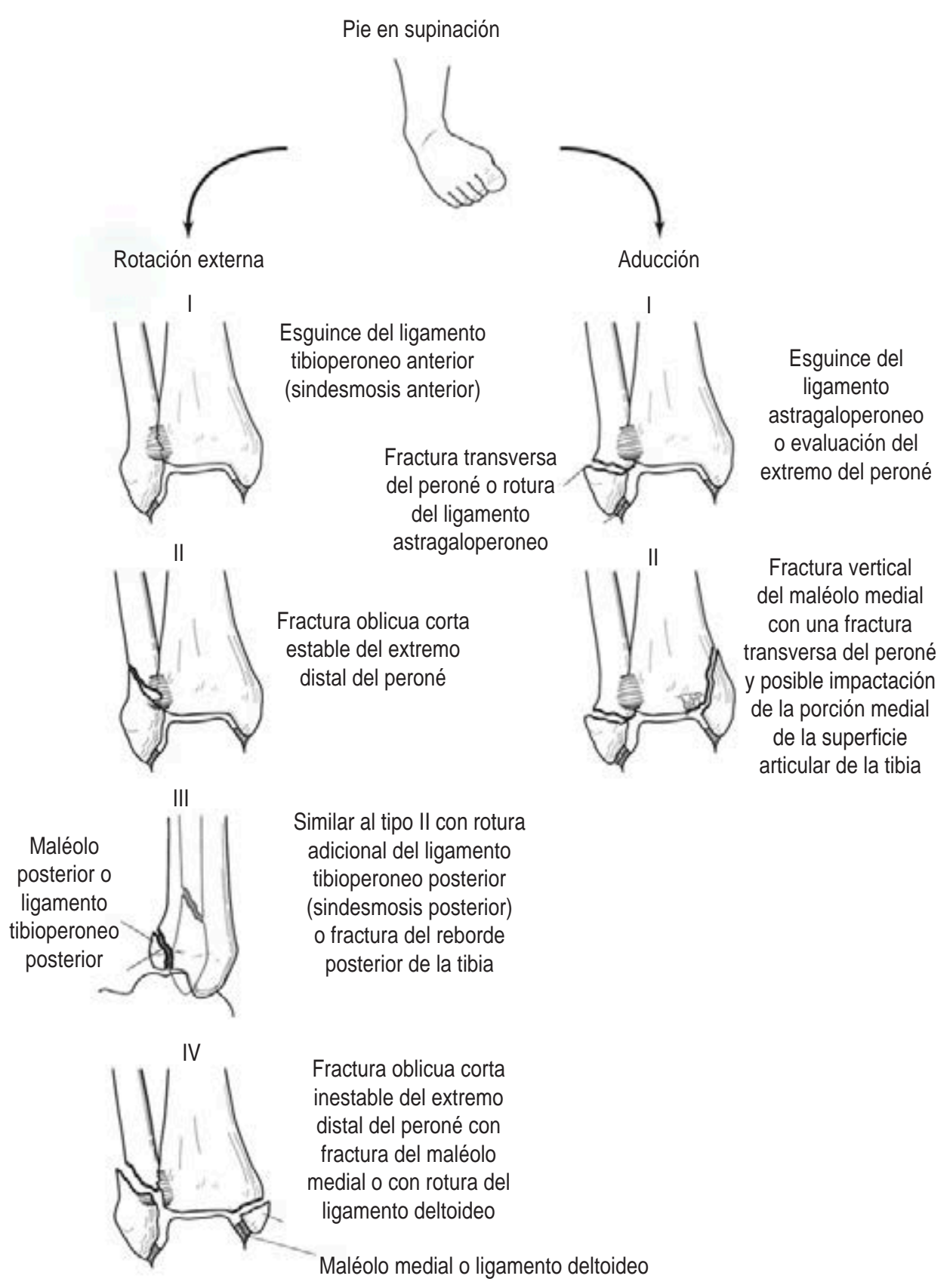

Figura 2:

Diagrama de clasificación de Lauge-Hansen de fracturas con mecanismo de supinaciónrotación externa y supinación-aducción. Adaptada de: Marsh J, Saltzman C. Ankle fractures. In: Buchloz R, Heckman J, eds. Rockwood and Green's fractures in adults. 5th ed. Philadelphia, PA: Lippincott Williams \& Wilkins; 2001. pp. 2001-2090.

equimosis y edema); sin embargo, nos podemos apoyar en la radiografía con estrés y con gravedad para evaluar la estabilidad medial a través de la apertura del claro medial, siendo una medición $>5 \mathrm{~mm}$ el estándar de oro para indicar ruptura del complejo ligamentario deltoideo.

El objetivo de este estudio es optar por un algoritmo de tratamiento no quirúrgico en aquellos pacientes con fracturas de maléolo lateral aislado tipo Weber $B$ estables con bota ortopédica neumática para así evitar el riesgo quirúrgico y anestésico, además de complicaciones como lesión nerviosa e infección del sitio quirúrgico, inmovilización prolongada y mayor tiempo de rehabilitación.

\section{Material y métodos}

Estudio transversal analítico en pacientes con fractura de maléolo tipo Weber B estables, a 
quienes se les colocó bota ortopédica neumática y se les evaluó funcionalidad en el Departamento de Traumatología y Ortopedia del Hospital Civil de Guadalajara «Fray Antonio Alcalde», Jalisco, México.

Los criterios de inclusión fueron pacientes adultos de 18 a 60 años de edad, de cualquier sexo, que acudieron a Urgencias del Hospital Civil de Guadalajara «Fray Antonio Alcalde» con fracturas de maléolo lateral aislado tipo Weber $B$ estables, de menos de una semana de evolución, en el periodo comprendido entre agosto de 2018 y agosto de 2019.

Los criterios de exclusión fueron pacientes menores de 18 años o mayores de 60 años de edad, fracturas mayores a una semana de evolución, pacientes con manejo previo fuera de la institución (fractura o cirugía previa del tobillo afectado), fracturas de maléolo bilaterales o inestables y aquellos pacientes que se nieguen a participar en el estudio y/o firmar el consentimiento informado.

Los criterios de salida fueron pacientes trasladados a otra unidad para su manejo o seguimiento, pacientes con mal apego al tratamiento $o$ a las indicaciones médicas y aquéllos que revocaron su participación en el estudio.

Los pacientes que ingresaron al estudio fueron manejados de manera conservadora con colocación de bota ortopédica neumática tipo Walker larga por seis semanas, se les permitió la descarga de peso inmediata y posterior a este periodo se cambió por tobillera con varilla, permitiendo actividades de bajo impacto hasta las 12 semanas, corte de tiempo en el que se eliminaron todas las restricciones de movimiento y se comenzó la adaptación regular a sus actividades normales.

Las mediciones de las escalas EVA (escala visual análoga), (Olerud molander ankle score) OMA, FAAM (Foot and Ankle Ability Measure) y AOFAS (American Orthopaedic Foot and Ankle Society) fueron realizadas por un médico estandarizado a los 12 meses del evento.

Una vez obtenida la muestra, se hizo la extracción de datos de las variables de interés mediante el instrumento de extracción en físico. Se realizó el vaciado de los datos del documento fuente a una base de datos electrónica codificada para el análisis de los datos y la obtención de los resultados.

El estudio no fue financiado por un patrocinador externo y fue aprobado por el Comité de Ética del hospital donde se realizó.

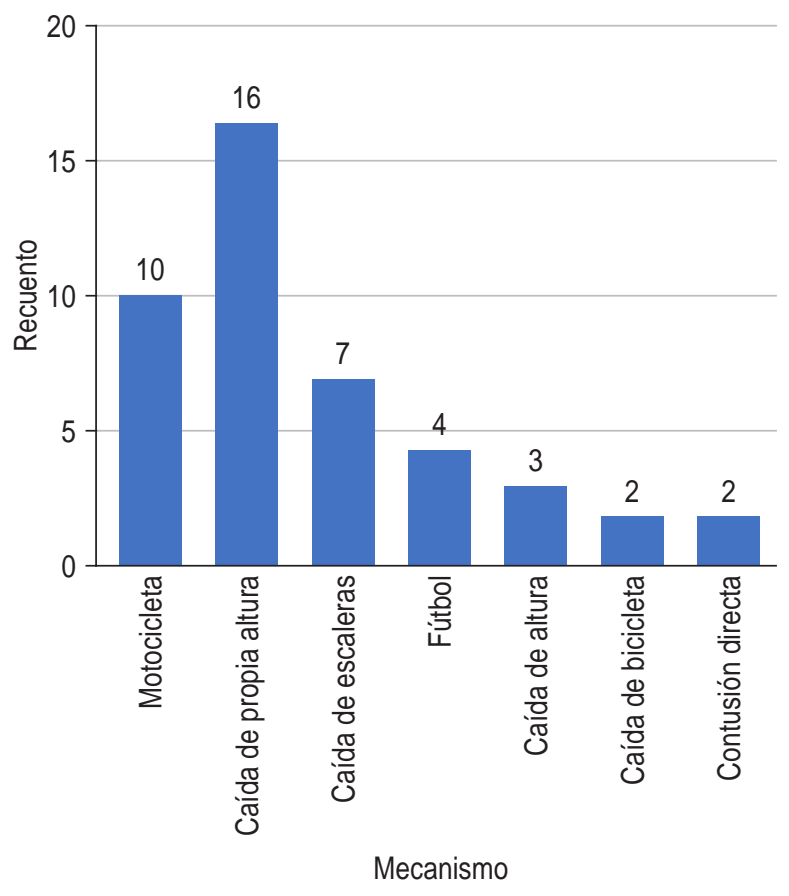

Figura 3: Distribución del mecanismo de acción en las fracturas de maléolo lateral.

\section{Resultados}

Se incluyeron 44 pacientes en el estudio y son los que se muestran en el análisis estadístico. La prueba de normalidad de Kolmogorov-Smirnov mostró una distribución simétrica de la población. El sexo masculino prevaleció en la muestra $(n=26,59.1 \%)$ frente al femenino $(n=18,40.9 \%)$. La media de edad fue de $34.7 \pm 13.2$ años.

Respecto a las variables demográficas de la muestra, la ocupación más frecuente fue ama de casa $(n=11,25 \%)$, seguido de empleado y comerciante ( $n=8,18.2 \%$ para cada uno). Trece punto seis por ciento reportó diabetes tipo $2(n=6), 9.1 \%$ hipertensión arterial $(n=4), 6.8 \%$ retraso del neurodesarrollo $(n=3), 4.5 \%$ epilepsia $(n=2)$ y $2.3 \%$ ceguera y parálisis facial $(n=1)$. El alcoholismo fue la toxicomanía más prevalente $(n=11,25 \%)$, seguido del tabaquismo $(n=8,18.2 \%)$ y el consumo de drogas $(n=2,4.5 \%)$.

El miembro dominante más frecuente fue el derecho $(n=41,93.2 \%)$. El tobillo derecho y el izquierdo mostraron la misma prevalencia de fractura $(n=22$, $50 \%$ para cada uno). El mecanismo de lesión más prevalente fue la caída de propia altura $(n=16$, $36.4 \%)$, seguido de accidente en motocicleta $(n=10$, 
22.7\%) (Figura 3). El tiempo de fractura a la atención más común fue de más de 72 horas $(n=23,52.3 \%)$, seguido de 24 a 48 horas $(n=8,18.2 \%)$, menos de 24 horas $(\mathrm{n}=7,15.9 \%)$ y por último de 48 a 72 horas $(\mathrm{n}=6,13.6 \%)$.

La satisfacción en la atención fue total en $88.6 \%$ de la muestra $(n=39)$, seguido de moderado $(n=3$, $6.8 \%)$ y poca satisfacción $(n=2,4.5 \%)$.

Respecto al dolor, la media de dolor evaluada por EVA fue de $2.6 \pm 2$. La mayor parte de la muestra no presentó dolor ( $n=18,40.9 \%)$, seguido de dolor ligero $(n=14,31.8 \%)$, moderado $(n=7,15.9 \%)$ y finalmente intenso $(n=5,11.4 \%)$.

En cuanto a la valoración por AOFAS, la media de puntuación fue de $89.6 \pm 12.4$ puntos. Se catalogó como funcional a $95.5 \%$ de la muestra $(n=42)$. La media de puntuación de FAAM fue de $80.6 \pm 9.4$ puntos y FAAM deportes con media de $25.8 \pm 5$. Respecto a OMA, la media de puntuación fue de $93.4 \pm 12.9$ puntos (Figura 4).

Respecto a la medición del claro medial inicial, el más prevalente fue $4(n=22,50 \%)$, seguido de 5 $(n=14,31.8 \%)$ y $6(n=8,18.2 \%)$. El claro medial final fue de $4(n=22,50 \%)$, seguido de $5(n=9,20.5 \%)$ y finalmente $3(n=13,29.5 \%)$.

La muestra se dividió en dos para su análisis: los pacientes a los que se les colocó bota y los que requirieron cirugía. Las características por grupo se enumeran en la Tabla 1.

EVA. Se observó una mayor puntuación en EVA $(t(42)=3.025, p=0.004)$ en el grupo quirúrgico (media $=4$ ) al compararlo con el grupo de bota (media $=1.4$ ). La cirugía se asoció con mayor dolor en forma estadísticamente significativa $(p=0.001$ ) (Figura 5).

AOFAS. Se observó una menor puntuación funcional en AOFAS ( $t(42)=-2.577, p=0.014$ ) en el grupo quirúrgico (media $=8.8$ ) al compararlo con el grupo de bota (media =9.8). Así como en la puntuación del retropié en AOFAS (t $(42)=-3.443$, $p=0.001)$ en el grupo quirúrgico (media $=4.7)$ en comparación con el grupo de bota (media $=6$ ). Sin embargo, no hubo diferencias estadísticas en la puntuación final.

Se evidenció una asociación estadísticamente significativa, moderada y directamente proporcional entre la intervención quirúrgica y la inestabilidad por AOFAS (ro $=0.499, p=0.001$ ). Los pacientes que reciben cirugía tienen 20 veces más probabilidades de cursar con inestabilidad frente a los que se les colocó bota (RR 20, IC 95\% 2.2-76.8). Asimismo,

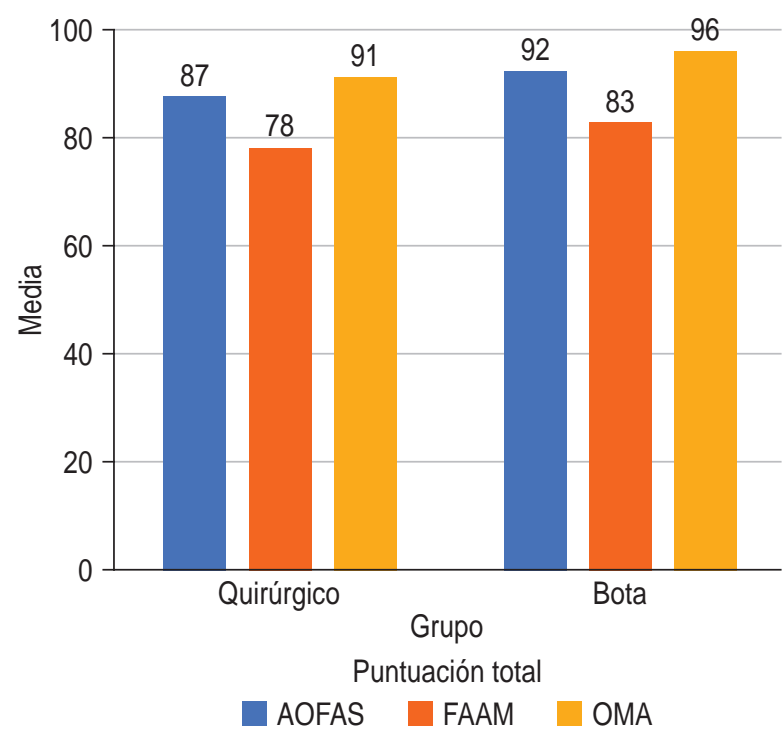

Figura 4: Distribución de resultados finales de tres escalas funcionales (AOFAS, FAAM, OMA) categorizada por grupo de estudio.

la cirugía se asoció con clasificación moderada en retropié $(p=0.003)$.

FAAM. No se evidenciaron diferencias entre la puntuación por escala FAAM entre los dos grupos.

OMA. Se observó una menor puntuación en edema por OMA (t $(42)=-3.238, p=0.002$ ) en el grupo quirúrgico (media $=5.7$ ) al compararlo con el grupo de bota (media $=8.4$ ). Así como menor puntuación en actividades diarias por OMA $(t(42)=-2.131, p=0.039)$ en el grupo quirúrgico (media $=18.5$ ) en comparación con el grupo de bota (media $=20$ ). Sin embargo, no hubo diferencias estadísticas en la puntuación final.

Claro medial. La medición del claro medial inicial es diferente $(z=-2.234, p=0.025)$ entre el grupo de bota (mediana $=4$ ) y el grupo quirúrgico (mediana $=5$ ). Se evidenció una asociación estadísticamente significativa entre el claro medial anormal inicial $(p=0.035)$ y el final anormal $(p=0.043)$ con el tratamiento quirúrgico.

\section{Discusión}

La evaluación y tratamiento de las fracturas de maléolo lateral tipo Weber B estables, a pesar de ser el tipo de fractura más común del tobillo, hasta la fecha, continúa controversial.

Aunque generalmente es aceptado que este tipo de fracturas requieren estabilización quirúrgica, cuando el paciente es debidamente seleccionado 
se puede optar por el tratamiento conservador con buenos resultados funcionales.

En este estudio se presentó una alternativa al tratamiento conservador convencional al introducir la colocación de una bota Walker neumática larga y permitir el apoyo del paciente desde el primer día de fracturado; esto con el objetivo de acortar el tiempo de rehabilitación al no existir atrofia muscular por desuso y permitir que el paciente continúe con actividades diarias y laborales al no estar dependiendo de muletas u otros aparatos ortopédicos.

Por otra parte, se propone la toma de radiografías iniciales con estrés (proyección en mortaja) y la subsecuente toma de radiografías seriadas anteroposterior y lateral de tobillo con descarga de peso (en bipedestación) como un método adecuado para diferenciar las fracturas que pueden tratarse de manera segura con manejo conservador de aquéllas que requieren fijación quirúrgica.

Recientemente, se ha cuestionado el uso de radiografías con descarga de peso en una investigación cadavérica; ${ }^{3}$ no obstante, un ensayo clínico reciente lo contradice. ${ }^{4}$ Aunque otros estudios imagenológicos se han propuesto, las radiografías simples siguen siendo las más utilizadas para evaluar las fracturas de tobillo por ser las más fáciles de obtener e interpretar. Sin embargo, se debe prestar atención al tomar la proyección de mortaja, la cual se realiza con el pie a $15-20^{\circ}$ de rotación interna para compensar el eje intermaleolar; cualquier cambio en la posición del pie puede afectar las mediciones del claro medial. En dicha proyección, un espacio de claro medial mayor a $5 \mathrm{~mm}$ se interpretó como anormal e indica un desplazamiento lateral del astrágalo, así como un solapamiento tibioperoneo mayor a $1 \mathrm{~cm}$ implica una ruptura franca de la sindesmosis.

En este estudio, se les realizaron las radiografías con descarga de peso para el seguimiento a todos los pacientes; cabe señalar que ninguno tuvo molestias o dificultad para tolerar la bipedestación en la primera cita de seguimiento (una semana); además, ninguno demostró ensanchamiento del claro medial en ninguna de las radiografías subsecuentes. Debido a esto, podríamos incluso considerar eliminar la segunda cita de seguimien-

Tabla 1: Características por grupo.

\begin{tabular}{|c|c|c|c|}
\hline Variable & $\begin{array}{c}\text { Bota }(\mathrm{N}=23) \\
\mathrm{n}(\%)\end{array}$ & $\begin{array}{c}\text { Quirúrgico ( } \mathrm{N}=21) \\
\mathrm{n}(\%)\end{array}$ & $\mathrm{p}$ \\
\hline Edad (años) ${ }^{*}$ & $37.2 \pm 9.9$ & $31 \pm 15.6$ & 0.118 \\
\hline Sexo & & & 0.802 \\
\hline Femenino & $9(39.1)$ & $9(42.9)$ & \\
\hline Masculino & $14(60.9)$ & $12(57.1)$ & \\
\hline Tiempo de fractura (h) & & & 0.054 \\
\hline$<24$ & $2(8.7)$ & $5(23.8)$ & \\
\hline 24 a 48 & $8(34.8)$ & $0(0)$ & \\
\hline 49 a 72 & $6(26.1)$ & $0(0)$ & \\
\hline$>72$ & $7(30.4)$ & $16(76.2)$ & \\
\hline$E V A^{*}$ & $1.43 \pm 1$ & $4 \pm 3.5$ & 0.004 \\
\hline AOFAS* & $92.2 \pm 7.7$ & $86.9 \pm 15.9$ & 0.162 \\
\hline FAAM $^{*}$ & $82.8 \pm 1.3$ & $78.2 \pm 13.3$ & 0.112 \\
\hline FAAM deportes* & $27 \pm 2.1$ & $24.5 \pm 6.7$ & 0.097 \\
\hline $\mathrm{OMA}^{*}$ & $95 \pm 5.9$ & $90.7 \pm 17.5$ & 0.191 \\
\hline Claro medial inicial & & & 0.025 \\
\hline 4 & $15(65.2)$ & $7(33.3)$ & \\
\hline 5 & $6(26.1)$ & $8(38.1)$ & \\
\hline 6 & $2(8.7)$ & $6(28.6)$ & \\
\hline Claro medial final & & & 0.107 \\
\hline 3 & $8(34.8)$ & $5(23.8)$ & \\
\hline 4 & $13(56.5)$ & $9(42.9)$ & \\
\hline 5 & $2(8.7)$ & $7(33.3)$ & \\
\hline
\end{tabular}

* Los valores son expresados en media \pm desviación estándar.

$\mathrm{EVA}=$ escala visual análoga; $\mathrm{AOFAS}=$ American Orthopaedic Foot and Ankle Society; FAAM = Foot and Ankle Ability Measure; OMA = Olerud molander ankle score. 


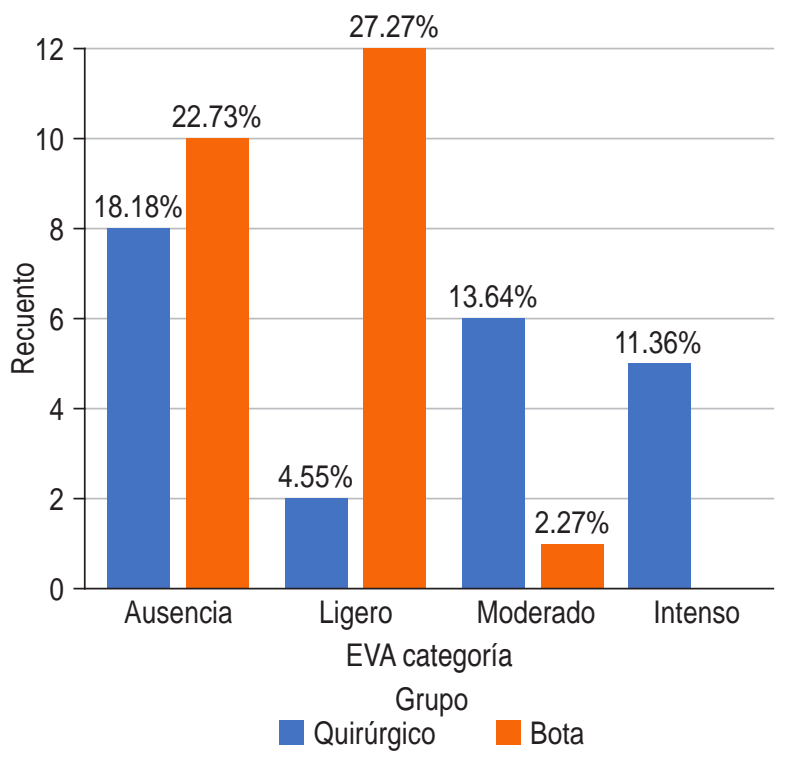

Figura 5: Distribución de la escala visual análoga categorizada por grupo de estudio.

to a las dos semanas de evolución del algoritmo de tratamiento.

Con estos hallazgos, podríamos también cuestionar el uso de la medición del claro medial mayor de $5 \mathrm{~mm}$ como punto de referencia para determinar si el paciente se opera o no; esto reforzado por un estudio que indica que la medición del claro medial podría variar de acuerdo con las características inherentes de la persona, como el sexo y la altura. ${ }^{5}$ La mayoría de los pacientes en este estudio reportaron resultados funcionales excelentes a pesar de la medición del claro medial.

Estos resultados son consistentes con un estudio aleatorizado reciente, ${ }^{6}$ el cual encontró buenos resultados funcionales en pacientes con claro medial $>5$ $\mathrm{mm}(5.8 \pm 1.1 \mathrm{~mm})$ tratados tanto de manera quirúrgica como no quirúrgica. En este mismo estudio, se observó retraso de la consolidación o no unión en ocho pacientes con claro medial $>5 \mathrm{~mm}$ en un grupo de 40 pacientes tratados no quirúrgicamente. En contraste, no encontramos ningún caso de no unión o de persistencia del ensanchamiento del claro medial $>5$ $\mathrm{mm}$ al año de seguimiento. Por otra parte, también se reportaron cinco pacientes con infección del sitio quirúrgico y cinco pacientes que requirieron el retiro del implante en un grupo de 41 pacientes tratados con cirugía, lo cual evidencia los riesgos potenciales que se pueden evitar si el procedimiento quirúrgico es innecesario en casos seleccionados. ${ }^{6}$
En nuestro estudio, no se observó tendencia que indicara que la medición de un claro medial inicial $>5$ $\mathrm{mm}$ en las radiografías con estrés fuera predictivo de un peor resultado funcional, lo cual contradice al estudio de Clements y colaboradores. ${ }^{7}$

Asimismo, con respecto a la medición del claro medial, todos los pacientes con radiografías con descarga de peso iniciales normales mantuvieron dicha alineación durante todo el curso del tratamiento. Por lo tanto, se puede postular que las radiografías con descarga de peso por sí mismas pueden evaluar satisfactoriamente la estabilidad medial en las fracturas Weber B.

Entre los resultados obtenidos, destacan la asociación estadísticamente significativa, moderada y directamente proporcional entre la intervención quirúrgica y la inestabilidad del retropié por AOFAS ( $\mathrm{ro}=0.499$, $\mathrm{p}=0.001$ ). Los pacientes que reciben cirugía tienen 20 veces más probabilidades de cursar con inestabilidad frente a los que se les colocó bota (RR 20, IC $95 \%$ 2.2-76.8). De igual manera, la cirugía se asoció con mayor dolor residual en forma estadísticamente significativa $(p=0.001)$.

Entre las fortalezas al realizar este estudio se incluye la naturaleza prospectiva con la cual se incorporaron los pacientes, lo cual optimizó la estandarización del cuidado y la recolección de datos. Este estudio se realizó en un solo centro hospitalario, permitiendo que sólo un médico (la autora principal) diera seguimiento personalmente a cada paciente, evitando así discrepancias en el análisis y la recolección de datos.

Por otro lado, el uso de varias escalas funcionales permitió describir ampliamente los resultados y evitar las deficiencias de cualquiera de dichos sistemas de puntuación.

Las limitaciones del estudio incluyen diseño retrospectivo del estudio, a pesar de que toda la información se recolectó prospectivamente y los pacientes fueron tratados consistentemente con los parámetros de los métodos del mismo. También, este estudio se encuentra limitado por el número relativamente pequeño de ambos grupos de pacientes. En adición a esto, un número significativo de pacientes no acudieron a su revisión de un año de seguimiento.

Para finalizar, a pesar de que los resultados a corto plazo en este estudio son prometedores, reconocemos que este tipo de fracturas requieren de seguimiento a largo plazo para determinar los resultados definitivos. 


\section{Conclusiones}

La colocación de bota ortopédica neumática se asocia a buena funcionalidad del tobillo en pacientes con fractura de maléolo lateral aislado tipo Weber B estable.

1. Se observó una menor puntuación funcional en AOFAS en el grupo quirúrgico al compararlo con el grupo de bota.

2. Se observó una mayor puntuación en EVA en el grupo quirúrgico en comparación con el grupo de bota.

3. Los pacientes que reciben cirugía tienen 20 veces más probabilidades de cursar con inestabilidad frente a los que se les colocó bota.

4. No se evidenciaron diferencias entre la puntuación por escala FAAM entre los dos grupos.

5. Se observó una menor puntuación en edema por OMA en el grupo quirúrgico al compararlo con el grupo de bota. Así como menor puntuación en actividades diarias por OMA en el grupo quirúrgico en comparación con el grupo de bota.

\section{Referencias}

1. Close JR. Some applications of the functional anatomy of the ankle joint. J Bone and Joint Surg. 1956; 38 (A): 761-781.

2. Makkozzay PTH. Complicaciones de las fracturas de tobillo. Ortho-tips. 2006; 2 (4): 262-269.

3. Stewart C, Saleem O, Mukherjee DP, Suk M, Marymont J, Anissian L. Axial load weightbearing radiography in determining lateral malleolus fracture stability: a cadaveric study. Foot Ankle Int. 2012; 33 (7): 548-552.

4. Hoshino CM, Nomoto EK, Norheim EP, Harris TG. Correlation of weightbearing radiographs and stability of stress positive ankle fractures. Foot Ankle Int. 2012; 33 (2): 92-98.

5. Murphy JM, Kadakia AR, Schilling PL, Irwin TA. Relationship amongradiographic ankle medial clear space, sex and height. Orthopedics. 2014; 37 (5): 449-454.

6. Sanders DW, Tieszer C, Corbett B; Canadian Orthopedic Trauma Society. Operative versus nonoperative treatment of unstable lateral malleolar fractures: A randomized multicenter trial. J Orthop Trauma. 2012; 26 (3): 129-134.

7. Clements JR, Motley TA, Garrett A, Carpenter BB. Nonoperative treatment of bimalleolar equivalent ankle fractures: a retrospective review of 51 patients. J Foot Ankle Surg. 2008; 47 (1): 40-45.

\section{Conflicto de intereses}

No se declara conflicto de intereses. 